\title{
Blood pressure control predicts plasma matrix metalloproteinase- 9 in diabetes mellitus type II
}

Katerina Vitlianova ${ }^{1}$, Janeta Georgieva ${ }^{2}$, Maria Milanova ${ }^{3}$, Svetlin Tzonev ${ }^{1}$

'Department of Internal Medicine, Clinic of Cardiology, Medical University of Sofia, Sofia, Bulgaria

2Department of Internal Medicine, Clinic of Cardiology, Medical University of Varna, Varna, Bulgaria

${ }^{3}$ Clinic of Cardiology and Internal Medicine, Institute of Emergency Medicine

"N.I. Pirogov" Sofia, Sofia, Bulgaria

Submitted: 28 October 2012

Accepted: 27 January 2013

Arch Med Sci 2015; 11, 1: 85-91

DOI: 10.5114/aoms.2015.49208

Copyright $\odot 2015$ Termedia \& Banach

\section{Abstract}

Introduction: Matrix metalloproteinase-9 (MMP-9) plays an important role in extracellular and vascular remodelling. We aimed therefore to assess the role of blood pressure (BP) control on plasma MMP-9 in relation to the presence of diabetes mellitus (DM) type II.

Material and methods: Plasma MMP-9 was measured in 61 patients who were divided into two groups depending on their BP control as follows: 49 patients with uncontrolled arterial hypertension (AH) defined as BP values >130/80 mm Hg and 12 patients with optimal blood pressure values. Plasma MMP-9 levels were measured with immunoassay at discharge. Group comparisons were made with the independent $t$-test, Mann-Whitney $U$-test and $\chi^{2}$ test where appropriate. The associations of the variables with MMP-9 were investigated with linear regression analyses.

Results: The diabetics made up $34.4 \%$ of the investigated patients. Frequency of DM did not differ between the two BP groups $(30.0 \%$ vs. $36.6 \%$, $p>0.05)$. Plasma MMP-9 concentrations differed significantly between the diabetics vs. non-diabetics (median: $1.9 \mathrm{ng} / \mathrm{ml}$, range: $1.0-7.3$ vs. $1.4 \mathrm{ng} / \mathrm{ml}$, range: $0.5-4.7, p<0.05)$. Stratification across the categories of BP control showed a significant correlation between plasma MMP-9 and DM type II only in the uncontrolled BP group. The significance of that relationship disappeared in the group of patients with optimal BP control.

Conclusions: Plasma values of MMP-9 are raised in patients with DM type II. The results revealed the impact of the combination of uncontrolled $\mathrm{AH}$ and DM type II on vascular remodelling processes.

Key words: matrix metalloproteinases, type II diabetes mellitus, arterial hypertension.

\section{Introduction}

Cardiovascular disease (CVD) and complications are the leading cause of morbidity and mortality in patients with type II diabetes mellitus (DM) [1]. Control of blood pressure (BP) plays an important part in the management of type II DM. Optimal BP control has a crucial impact on improvement of both prognosis and prevention of complications in diabetics, whereas undertreatment of BP and failure to achieve the recommended target values constitute a considerable and acknowledged

\author{
Corresponding author: \\ Katerina Vitlianova \\ Department \\ of Internal Medicine \\ Clinic of Cardiology \\ Medical University of Sofia \\ 1 St Georgi Sofiiski St \\ Alexandrovs University \\ Hospital \\ 1431 Sofia, Bulgaria \\ Phone: +3592888553451 \\ E-mail: k.vitlianova@abv.bg
}


problem worldwide. The basic mechanisms of that protection are related to the vascular alterations and extracellular remodelling but are not fully understood and clarified yet [2].

Over the last decade, considerable evidence has pointed out the role of matrix metalloproteinases (MMPs) in regulation of vascular remodelling [3-6]

The major MMP species in the myocardium and vasculature that have been most frequently related to myocardial and vascular matrix remodelling are represented by gelatinases consisting of two main types, MMP-2 and MMP-9 [7]. Several studies have documented downregulation of MMP-9, explaining the observed mesangial matrix expansion found in diabetic nephropathy [8-10]. However, recent studies have demonstrated increased MMP9 renal and aortic expression in diabetes [11, 12]. Increased circulating MMP-9 levels have also been observed in subjects with systemic hypertension [13] or isolated systolic hypertension [14].

Whether and to what degree diabetes affects the expression and activity of the MMPs in patients with arterial hypertension remains unknown.

There are limited data on the complex relationship between MMP-9, values of BP and DM type II, having in mind the shared common basic mechanism of pathological vascular remodelling in both conditions. The growing interest in MMP-9 is also related to the suggestions that the induction of the vascular MMP system may be a potential target for extracellular matrix (ECM) regulation by cytokines and growth factors in diabetes, with therapeutic implications for management of patients with DM type II.

We aimed, therefore, to assess the role of BP control on plasma MMP-9 in relation to the presence of DM type II.

\section{Material and methods}

\section{Patient population and study protocol}

Plasma MMP-9 levels were measured in 61 patients aged (mean \pm SD) $69.9 \pm 7.5$ years, body mass index (BMI) $30.1 \pm 5.8 \mathrm{~kg} / \mathrm{m}^{2}, 44.3 \%$ women, diabetics $34.4 \%$, age 71.6 years, BMI 30.1 $\pm 6.1 \mathrm{~kg} / \mathrm{m}^{2}$, admitted to the Clinic of Cardiology, Department of Internal Medicine, University Hospital Alexandrovska, Medical University of Sofia over the 6-month period from 1 May, 2010. Cases were eligible for inclusion in the study if they were older than 18 years and had been diagnosed as hypertensives based on patient history and clinical examination. Patients having suffered acute myocardial infarction (AMI) or stroke within three months and any disease of less than a 1-year period of expected survival, or severe disability due to any cause, were excluded. Ischaemic heart disease (IHD) was defined in the case of angiographic con- firmation (37.7\%) and/or history of the coronary event and angina pectoris with the corresponding electrocardiographic (ECG) and clinical pattern.

Patients were divided into two groups depending on their BP control in accordance with the Seventh Report of the Joint National Committee on Prevention, Detection, Evaluation, and Treatment of High Blood Pressure (JNC 7) recommendations as follows: 49 patients with uncontrolled arterial hypertension (AH) defined as BP values > 130/80 $\mathrm{mm} \mathrm{Hg}$ and 12 patients with optimal blood pressure values [15].

Diabetes mellitus type II was defined as fasting plasma glucose $\geq 7.0 \mathrm{mmol} / \mathrm{l}$ and glycated hemoglobin $\left(\mathrm{HbA}_{1 c}\right) \geq 6.5 \%$ according to the definitions of the World Health Organization and the American Diabetes Association (2010) [16, 17].

The study was approved by the local Ethical Committee at the Medical University of Sofia. Informed consent was obtained from the subjects before they were recruited into the study.

All patients underwent a detailed clinical examination and interview according to the standard protocol, to collect information on biologic and demographic data including age, gender, cardiovascular risk factors and blood pressure (systolic and diastolic). All information on medical history, comorbidities, clinical characteristics and medications was documented.

Instrumental assessment was conducted at admission and included standard electrocardiogram obtained from 12-lead and transthoracic echocardiography. Echocardiography was undertaken with the patient in the left lateral decubitus position using a 3.5 MHz transducer and Phillips HD 11XE equipment. Standard M-mode was applied for obtaining left ventricle dimensions including left ventricular internal diameter. Interventricular septum thickness and posterior wall thickness were measured at the end of the diastole. Left ventricular hypertrophy (LVH) was assessed by calculating the left ventricular mass (LVM). Left ventricular mass was calculated according to the Devereux formula. Left ventricular mass index (LVMI) was obtained by dividing the LVM by body surface area. Left ventricular hypertrophy was defined as LVMI $>134 \mathrm{~g} / \mathrm{m}^{2}$ for men and $>110 \mathrm{~g} / \mathrm{m}^{2}$ for women [18].

\section{Biochemical tests}

Blood samples for standard biochemical analyses were taken at admission. Plasma MMP-9 levels were measured with immunoassay at discharge from the clinic. Blood samples were collected with a Vacutainer (Becton Dickinson, NJ, USA) in EDTA containing tubes. Samples were centrifuged up to $1 \mathrm{~h}$ after sampling and were stored at $-20^{\circ}$ until assayed. Blood investigations included information on blood count, serum electrolytes 
(sodium and potassium), creatinine, enzymes and lipid status.

The MMP-9 was determined using the available MMP-9 kit, IBL Hamburg. The concentration of plasma MMP-9 protein were investigated with enzyme immunoassay (EIA-ELISA assays) on a Trinitron ELISA reader in the Institute of Biology and Immunology of Reproduction at the Bulgarian Academy of Sciences, Sofia, Bulgaria. The sensitivity of the Human MMP-9 ELISA assay has been determined to be $0.05 \mathrm{ng} / \mathrm{ml}$. The intra-assay coefficient of variation of the MMP-9 ELISA test has been determined to be $7.3 \%$. The inter-assay coefficient of variation of the MMP-9 kit has been determined to be $<10 \%$.

All other serum parameters were measured as part of routine clinical testing in the central laboratory of the ALexandrowska University Hospital.

\section{Statistical analysis}

The data are summarised in terms of frequencies and percentages for categorical variables, and by means and standard deviations (SD) for continuous variables. Normally distributed variables are reported with the mean and skewed variables with the median and range of values. Group comparisons were made with the independent $t$-test, Mann-Whitney $U$-test and $\chi^{2}$ test where appropriate. Univariate associations of the variables with MMP-9 were investigated with linear regression analyses. Natural logarithmic transformation was used for skewed variables, including the dependent variable MMP-9. Multiple linear regression was used to investigate the independent relations to MMP-9. Multivariate linear regression models were conducted including the variables with a univariate level of significance of $p<0.010$. In all analyses, statistical significance was considered achieved at a value of $p \leq 0.05$.

Systolic blood pressure (SBP), diastolic blood pressure (DBP) and parameters of the lipid status, total serum cholesterol (TSC), high-density lipoprotein cholesterol (HDL-C), low-density lipoprotein cholesterol (LDL-C) and triglycerides were entered as continuous independent variables in linear regression analyses. The rest of the investigated independent variables were entered in the models as categorical variables.

The independent laboratory variables including parameters of blood count, creatinine and sodium were also measured on a continuous measurement scale.

Independent categorical variables included age, gender, DM type II, IHD, presence of chronic atrial fibrillation (AF) and history of myocardial infarction (MI) and therapy. Reference categories for the set of independent categorical variables were defined as age $\leq 65$ years, male gender, non-diabetics, non-ischaemic, sinus rhythm, and no treatment with inhibitors of angiotensin-converting enzyme/ angiotensin receptor (ACE/ARB) and $\beta$-blockers, statins and calcium channel blockers (CCB).

\section{Statistical analysis}

Statistical analyses were done using SPSS version 13.0 (SPSS Inc, Chicago, IL).

\section{Results}

Characteristics of the investigated patients are listed in detail in Tables I and II.

Median plasma MMP-9 in the study sample was $1.5 \mathrm{ng} / \mathrm{ml}$ (range: 0.5-7.3). Subjects with non-optimal antihypertensive control represented $32.8 \%$ (20) of the patients. Almost one third (21, 34.4\%) of the investigated patients were type II diabetics. Frequency of DM type II did not differ significantly between the two BP groups (36.6\% vs. $30.0 \%$, $p>0.05)$. Comparisons in demographic, clinical and biochemical data between the two groups (diabetics vs. non-diabetics) are presented in Tables III and IV. Diabetics had significantly higher plasma MMP-9 in comparison to non-diabetics.

Table I. Characteristics of patients $(n=61)$ investigated for MMP-9

\begin{tabular}{|c|c|}
\hline Parameter & Patients, $n(\%)$ \\
\hline \multicolumn{2}{|c|}{ Comorbidities and risk factors: } \\
\hline COPD & $10(16.9)$ \\
\hline PVD & 7 (11.9) \\
\hline Smoking & $8(13.6)$ \\
\hline IHD & $27(44.3)$ \\
\hline Myocardial infarction & $22(36.1)$ \\
\hline Angiography & $23(37.7)$ \\
\hline \multicolumn{2}{|l|}{ Clinical signs: } \\
\hline Chronic atrial fibrillation & $19(36.1)$ \\
\hline $\mathrm{SBP}[\mathrm{mm} \mathrm{Hg}]$ & $133.9(21.6)$ \\
\hline $\mathrm{DBP}[\mathrm{mm} \mathrm{Hg}]$ & $80.3(12.3)$ \\
\hline \multicolumn{2}{|l|}{ Instrumental data: } \\
\hline LVMI $\left[\mathrm{g} / \mathrm{m}^{2}\right]$ & $142.6(47.1)$ \\
\hline \multicolumn{2}{|l|}{ Therapy at admission: } \\
\hline ACE/ARB & $38(62.3)$ \\
\hline$\beta$-Blockers & $38(62.3)$ \\
\hline $\mathrm{CCB}$ & $8(13.1)$ \\
\hline Statins & $18(29.5)$ \\
\hline
\end{tabular}

COPD - chronic obstructive pulmonary disease, PVD - peripheral vascular disease. 
Table II. Laboratory characteristics of patients $(n=61)$ investigated for MMP-9

\begin{tabular}{|lc|}
\hline Parameter & Patients \\
\hline MMP-9 $[\mathrm{ng} / \mathrm{ml}]$ & $1.5(0.5-7.3)$ \\
\hline TSC $[\mathrm{mmol} / \mathrm{l}]$ & $4.7(1.0)$ \\
\hline Fasting blood glucose $[\mathrm{mmol} / \mathrm{l}]$ & $5.8(3.6-16)$ \\
\hline Haemoglobin $[\mathrm{g} / \mathrm{l}]$ & $126.9(19.1)$ \\
\hline Creatinine $[\mu \mathrm{mol} / \mathrm{l}]$ & $126.5(44.0)$ \\
\hline Sodium $[\mathrm{mmol} / \mathrm{l}]$ & $142(127-146)$ \\
\hline Leucocytes $\left(\times 10^{9} / \mathrm{l}\right)$ & $7.6(2.1)$ \\
\hline
\end{tabular}

Values are presented as means with SD and medians with range, depending on the type of data.

The two groups differed significantly in the frequency of reported IHD and past history of MI as well as in the serum levels of triglycerides and fasting blood glucose. No differences were also observed for the frequency of prescription of the main types of drugs between the groups. Diabetics from the uncontrolled BP group more frequently had IHD ( 83.3 vs. $14.3, p=0.007)$ and $\mathrm{MI}(66.7 \%$ vs. $7.1 \%, p=0.014)$ and were more frequently treated with $\beta$-blockers ( $100.0 \%$ vs. $41.7 \%, p=0.038)$ as compared to the non-diabetics. Significant differences in the distribution of the investigated factors according to diabetic status within the controlled BP group were not detected.

The results from the univariate linear regression analysis showed that type II DM, presence of $\mathrm{AF}$ and therapy with $\beta$-blockers are significant predictors of plasma MMP-9 levels in our study (Table $\mathrm{V}$ ). None of the other variables investigated in our study, including laboratory variables, reached statistical significance in the conducted univariate analyses.

Independent predictors of plasma MMP-9 are shown in Table VI. Using the enter method, a significant model emerged $(F=4.51, p=0.003)$. Adjusted $R^{2}=0.206$.

Table III. Distribution of the investigated demographic and clinical data by diabetic status

\begin{tabular}{|c|c|c|c|}
\hline Parameter & $\begin{array}{c}\text { Diabetics }(N=21) \\
n(\%)\end{array}$ & $\begin{array}{c}\text { Non-diabetics }(N=40) \\
n(\%)\end{array}$ & Value of $p^{*}$ \\
\hline Age $\geq 65$ & $17(81.0)$ & $28(70.0)$ & NS \\
\hline Female gender & $10(47.6)$ & $17(42.5)$ & NS \\
\hline \multicolumn{4}{|c|}{ Comorbidities and risk factors: } \\
\hline COPD & $4(19.0)$ & $7(17.5)$ & NS \\
\hline PVD & $4(19.0)$ & $3(7.5)$ & NS \\
\hline CVD & $2(9.5)$ & $8(20.0)$ & NS \\
\hline Smoking & $2(9.5)$ & $6(15.0)$ & NS \\
\hline $\mathrm{IHD}$ & $15(71.4)$ & $12(30.0)$ & 0.003 \\
\hline Myocardial infarction & $12(57.1)$ & $10(25.0)$ & 0.024 \\
\hline Angiography & $10(47.6)$ & $13(32.5)$ & NS \\
\hline \multicolumn{4}{|l|}{ Clinical signs: } \\
\hline Chronic atrial fibrillation & $7(33.3)$ & $18(45.0)$ & NS \\
\hline $\mathrm{SBP}[\mathrm{mm} \mathrm{Hg}]$ & $137.4(18.9)$ & $132.1(22.9)$ & NS \\
\hline $\mathrm{DBP}[\mathrm{mm} \mathrm{Hg}]$ & $80.2(10.8)$ & $80.4(13.2)$ & NS \\
\hline BP control & $15(71.4)$ & $26(65.0)$ & NS \\
\hline \multicolumn{4}{|l|}{ Instrumental data: } \\
\hline LVMI $\left[\mathrm{g} / \mathrm{m}^{2}\right]$ & $138.5(29.4)$ & $144.7(54.7)$ & 0.045 \\
\hline \multicolumn{4}{|l|}{ Therapy at admission: } \\
\hline ACE/ARB & $12(60.0)$ & $18(48.6)$ & NS \\
\hline$\beta$-Blockers & $11(55.0)$ & $14(37.8)$ & NS \\
\hline$\overline{C C B}$ & $4(20.0)$ & $4(10.8)$ & NS \\
\hline Statins & $7(33.3)$ & $11(29.7)$ & NS \\
\hline
\end{tabular}


Table IV. Biochemical tests by diabetic status

\begin{tabular}{|c|c|c|c|}
\hline Parameter & Diabetics $(N=21)$ & Non-diabetics $(N=40)$ & Value of $p^{*}$ \\
\hline MMP-9 [ng/ml] & $1.9(1.0-7.3)$ & $1.4(0.5-4.7)$ & 0.049 \\
\hline $\mathrm{TSC}[\mathrm{mmol} / \mathrm{l}]$ & $4.8(1.0)$ & $4.7(1.1)$ & NS \\
\hline $\mathrm{HDL}-\mathrm{C}[\mathrm{mmol} / \mathrm{l}]$ & $1.2(0.2)$ & $1.4(0.4)$ & NS \\
\hline LDL-C [mmol/l] & $2.7(0.7)$ & $2.8(0.9)$ & NS \\
\hline Triglycerides [mmol/l] & $2.2(1.2)$ & $1.3(0.6)$ & 0.005 \\
\hline Fasting blood glucose $[\mathrm{mmol} / \mathrm{l}]$ & $7.5(4.6-16.0)$ & $5.7(3.6-7.9)$ & 0.001 \\
\hline Haemoglobin $[\mathrm{g} / \mathrm{l}]$ & $124.2(14.9)$ & $128.4(21.1)$ & NS \\
\hline Creatinine $[\mu \mathrm{mol} / \mathrm{l}]$ & $131.9(37.8)$ & $123.5(47.4)$ & NS \\
\hline Sodium $[\mathrm{mmol} / \mathrm{l}]$ & $141.5(136-146)$ & $142(127-146)$ & NS \\
\hline Leucocytes $\left[\times 10^{9} / \mathrm{I}\right]$ & $8.2(2.3)$ & $7.3(1.9)$ & NS \\
\hline
\end{tabular}

*Mann-Whitney U-test or T-test depending on type of data; NS - not significant.

Table V. Significant predictors for plasma MMP-9: univariate linear regression analysis

\begin{tabular}{|lccc|}
\hline Variable & $R^{2}$ & $\beta$ & Value of $p$ \\
\hline DM type II & 0.131 & 0.43 & 0.005 \\
Constant & & 0.34 & $<0.001$ \\
\hline AF (chronic) & 0.07 & -0.306 & 0.044 \\
Constant & & 0.61 & $<0.001$ \\
\hline$\beta$-Blockers & 0.094 & 0.35 & 0.023 \\
Constant & & 0.32 & 0.002 \\
\hline
\end{tabular}

Stratification across the categories of BP control showed a significant correlation between plasma MMP-9 and DM type II only in the uncontrolled BP group. The significance of that relationship disappeared in the group of patients with optimal BP control (Table VII).

\section{Discussion}

Despite the multitude data on the potential relationship between MMPs and diabetes, the exact clinical profile of plasma MMP-9 in hypertensive diabetic patients has not been thoroughly investigated. Our results revealed a significant relationship between the plasma MMP-9 and type II DM that changes and more specifically attenuates in conditions of optimal BP. The results pointed out the possible synergic effect of the two main cardiovascular conditions, type II DM and hypertension, on vascular remodelling processes in hypertensive dia-
Table VI. Predictive factors for plasma MMP-9: multiple linear regression analysis

\begin{tabular}{|lcc|}
\hline Factor & $\beta$ & Value of $p$ \\
\hline Constant & 0.31 & 0.020 \\
\hline DM type II & 0.35 & 0.025 \\
\hline IHD & 0.12 & NS \\
\hline AF & -0.28 & NS \\
\hline$\beta$-Blockers & 0.22 & NS \\
\hline
\end{tabular}

betic patients. It could be therefore considered that the basic mechanism of BP control protection goes through the delay of the already ongoing intensive vascular remodelling processes in type II DM. On the other hand, the observed significant differences in the frequency of IHD and $\beta$-blocker therapy according to the diabetic status in the uncontrolled group could contribute to the observed results through the known relationships of both factors to plasma MMP-9 reported in different studies.

The study also demonstrated higher plasma MMP-9 in subjects with type II DM as compared to non-diabetics. Current literature data on this issue are conflicting, with some studies reporting, similar to our results, raised plasma concentrations of MMP-9 [19, 20], while some studies demonstrated either no difference in plasma MMP-9 [21] or lower concentrations in patients with type II DM [22].

Table VII. Predictive effect of DM type II on MMP-9 across the BP groups

\begin{tabular}{|lcccccc|}
\hline Variable & \multicolumn{3}{c}{ BP uncontrolled group } & \multicolumn{3}{c|}{ BP controlled group } \\
\cline { 2 - 7 } & $R^{2}$ & $\beta$ & Value of $p$ & $R^{2}$ & $\beta$ & Value of $p$ \\
\hline Constant & & 0.28 & NS & & 0.36 & $<0.001$ \\
DM type II & 0.37 & 0.95 & 0.006 & 0.04 & 0.21 & NS \\
\hline
\end{tabular}


The results also correspond with the increased MMP-9 levels reported by Uemara et al. in animal models. In the paper by Dobos et al., the differences in results between the different studies were explained by differences in the type of study (human or experimental) and duration of diabetes. In the same article it was suggested that the changes in MMP synthesis and activity might be time-dependent, with upregulation of the MMP system in the early phase of diabetes, allowing the smooth muscle cells to migrate and contribute to intimal hyperplasia, whereas with the progression of diabetes, the MMP system is suppressed, causing ECM deposition and fibrosis [23, 24].

Evidence for the relationship between plasma MMP-9, type II DM and BP observed in our study comes from experimental studies showing a fall in plasma MMP-9 in close relation to the change in blood pressure which corresponds to the observed effect of BP control on the relationship between MMP-9 and DM type II. It has been established that $\mathrm{AH}$ is related to higher MMP-9 levels but optimal antihypertensive treatment could alter the levels towards a lower concentration as compared to non-treated individuals, which is in accordance with the difference in the relationship between MMP-9 and DM type II across the categories of the variable "BP control" observed in our study [25].

In support to our results are the observations that circulating MMP-9 levels are raised in treated hypertensive patients with type II DM, compared with normotensive control subjects after 1-year $\mathrm{CV}$ risk reduction therapy, measured as changes in MMP-9 levels in a similar high-risk population [26].

Close to the explanation of our results are the observations in hypertensive patients showing increase MMP-9 activity could result in increased degradation of elastin corresponding with the subsequent vessels' non-elasticity. In parallel to the observations on MMP-9 activity is the described decreased TIMP-1 activity that has been proved to lead to accumulation of poorly cross-linked, immature and unstable fibril degradation products, which result in misdirected deposition of collagen. Increased central and peripheral artery stiffness [27] occurs with diabetes, and higher circulating levels of MMP-9 complement the hypothesis that altered MMP- 9 activities could be linked to arterial stiffness [28].

Another point for consideration is the role of medications with angiotensin-converting enzyme inhibitors, and/or calcium channel blockers and/ or $\beta$-blockers.

All of these medications have been reported to lower MMP activity. The observed relationship between MMP-9 and $\beta$-blockers in the univariate analysis in our study is in accordance with the observations of Sezaki et al. in animal models that $\beta$-blockade prevents sustained metalloproteinase activation and diastolic stiffness induced by angiotensin II [29]. None of the mentioned types of drugs succeeded in changing the investigated relationship between the plasma MMP-9, DM and BP, which could be explained by the absence of significant treatment differences between the diabetic and non-diabetic group.

The major limitation of our study is the nontissue-based MMP-9 measure, which is difficult to implement, venesection being the most accessible analysis. The study results show that further work on vascular compliance in hypertensive diabetics is clearly warranted. Our modest sample size may have afforded limited power to detect potentially weaker correlations. Since this study was cross-sectional, we cannot draw conclusions on a proposed cause and effect relation between MMP-9, hypertension and DM type II. The generalisability of our findings also needs to be confirmed. We also do not have information on plasma MMP-9 as a prognostic factor in the selected study sample, and this should be addressed in future research. Furthermore, the gene expression was not measured in parallel to plasma levels, and the current study could not describe the pattern of induction of the enzyme but only describes the pattern of plasma profile in hypertensive patients with DM type II vs. non-diabetics. The study is also limited due to the single measurement of MMP9; there is a necessity for serial measurements of MMP-9 over the changing BP and diabetic status considering the above-discussed possibility for time-dependent relationships.

In conclusion, our data support the biologically plausible role of MMP-9, directed towards restriction of pathologic processes in hypertensive patients with DM type II. Plasma values of MMP-9 are increased in hypertensive patients with DM type II. The effect of DM on plasma MMP-9 depends on the quality of BP control. The results revealed the impact of the combination of uncontrolled arterial hypertension and presence of DM type II on the vascular remodelling processes, suggested by the observed changes in the relationship between MMP-9 and DM type II according to the BP category. The mechanisms of protection of optimal BP in diabetics are closely related to MMP-9 regulation, and the latter is a point for further investigations. The results revealed again the importance of $\mathrm{BP}$ control in diabetics and support the hypotheses of the role and acceleration of abnormal vascular remodelling in $\mathrm{DM}$ and $\mathrm{AH}$.

\section{Conflict of interest}

The authors declare no conflict of interest. 


\section{References}

1. Hein KD, King GL. Vascular abnormalities in diabetes mellitus. In: Contemporary endocrinology: endocrinology of the vasculature. Sowers JR (ed.). Humana Press, Totowa 1996.

2. Rysz J, Banach M, Stolarek RA, et al. Serum metalloproteinases MMP-2, MMP-9 and metalloproteinase tissue inhibitors TIMP-1 and TIMP-2 in patients on hemodialysis. Int Urol Nephrol 2011; 43: 491-8.

3. Spinale FG. Matrix metalloproteinases. Regulation and dysregulation in the failing heart. Circ Res 2002; 90: 520-30.

4. Thomas CV, Coker ML, Zellner JL, et al. Increased matrix metalloproteinase activity and selective upregulation in LV myocardium from patients with end-stage dilated cardiomyopathy. Circulation 1998; 97: 1708-15.

5. Banfi C, Cavalca V, Veglia F, et al. Neurohormonal activation is associated with increased levels of plasma matrix metalloproteinase-2 in human heart failure. Eur Heart J 2005; 26: 481-8.

6. Spinale FG, Coker ML, Krombach SR, et al Matrix metalloproteinase inhibition during the development of congestive heart failure: effects on left ventricular dimensions and function. Circ Res 1999; 85: 364-76.

7. Shah PK. Plaque disruption and thrombosis. Potential role of inflammation and infection. Cardiol Rev 2000; 1: 31-9.

8. Barylski M, Małyszko J, Rysz J, et al. Lipids, blood pressure, kidney - what was new in 2011? Arch Med Sci 2011; 7: 1055-66.

9. Rysz J, Banach M, Stolarek RA, et al. Serum matrix metalloproteinases MMP-2 and MMP-9 and metalloproteinase tissue inhibitors TIMP-1 and TIMP-2 in diabetic nephropathy. J Nephrol 2007; 20: 444-52.

10. Gambaro G, Nosadini R. Down-regulation of glomerular matrix metalloproteinase-2 gene in human NIDDM. Diabetologia 1997; 40: 1449-54.

11. Romanic AM, Burns-Kurtis CL, Ao Z, et al. Upregulated expression of human membrane type- 5 metalloproteinases in kidneys from diabetic patients. Am J Physiol Renal Physiol 2001; 281: F309-17.

12. Uemura S, Matsushita $\mathrm{H}$, Li W, et al. Diabetes mellitus enhances vascular matrix metalloproteinase activity. Circ Res 2001; 88: 1291-8.

13. Tayebjee MH, Nadar SK, MacFadyen RJ, Lip GY. Tissue inhibitor of metalloproteinase-1 and matrix metalloproteinase-9 levels in patients with hypertension relationship to tissue Doppler indices of diastolic relaxation. Am J Hypertens 2004; 17: 770-4.

14. Yasmin, McEniery CM, Wallace $S$, et al. Matrix metalloproteinase-9 (MMP-9), MMP-2, and serum elastase activity are associated with systolic hypertension and arterial stiffness. Arterioscler Thromb Vasc Biol 2005; 25: 372.

15. Seventh Report of the Joint National Committee on Prevention, Detection, Evaluation, and Treatment of High Blood Pressure.The National High Blood Pressure Education Program Coordinating Committee. Hypertension 2003; 42: 1206-52.

16. Definition and diagnosis of diabetes mellitus and intermediate hyperglycemia: report of a WHO/IDF consultation. World Health Organization, Geneva 2006; 21.

17. American Diabetes Association. Diagnosis and classification of diabetes mellitus. Diabetes Care 2010; 33 (Suppl. 1): S62-9.

18. Lang RM, Bierig M, Devereux RB, et al. Recommendations for chamber quantification: a report from the
American Society of Echocardiography's Guidelines and Standards Committee and the Chamber quantification Writing Group, developed in conjunction with the European Association Echocardiography, a branch of the European Society of Cardiology. I Am Soc Echocardiogr 2005; 18: 1440-63.

19. Signorelli SS, Malaponte G, Libra M, et al. Plasma levels and zymographic activities of matrix metalloproteinases 2 and 9 in type II diabetics with peripherial arterial disease. Vasc Med 2005; 10: 1-6.

20. Derosa G, D'Angelo A, Tinelli C, et al. Evaluation of metalloproteinase 2 and 9 levels and their inhibitors in diabetic and healthy subjects. Diabetes Metab 2007; 33: 129-34.

21. Papazafiropoulou A, Perrea D, Moyssakis I, et al. Plasma levels of MMP-2, MMP-9 and TIMP-1 are not associated with arterial stiffness in subjects with type 2 diabetes mellitus. J Diabet Comp 2010; 24: 20-7.

22. Sampson M, Davies I, Gavrilovic J, et al. Plasma matrix metalloproteinases, low density lipoprotein oxidisability and soluble adhesion molecules after a glucose load in type 2 diabetes. Cardiovasc Diabetol 2004; 3: 7-14.

23. Uemura S, Matsushita H, Li W, et al. Diabetes mellitus enhances vascular matrix metalloproteinase activity. Circ Res 2001; 88: 1291-8.

24. Portik-Dobos V, Anstadt MP, Hutchinson J, et al. Evidence for a matrix metalloproteinase induction/activation system in arterial vasculature and decreased synthesis and activity in diabetes. Diabetes 2002; 51: 3063-8.

25. Dandona P, Aljada A, Mohanty P, et al. Insulin suppresses plasma concentration of vascular endothelial growth factor and matrix metalloproteinase-9. Diabetes Care 2003; 26: 3310-4.

26. Tayebjee M, Lim H, MacFadyen R, Lip GY. Matrix metalloproteinase- 9 and tissue inhibitor of metalloproteinase- 1 and -2 in type 2 diabetes. Effect of 1 year's cardiovascular risk reduction therapy. Diabet Med 2001; 18: 777-80.

27. Schram M, Henry R, Van Dijk R, et al. Increased central artery stiffness in impaired glucose metabolism and type 2 diabetes: the Hoorn Study. Hypertension 2004; 43: 176-81.

28. Kontopoulos A, Athyros V, Pehlivanidis A, et al. Longterm treatment effect of atorvastatin on aortic stiffness in hypercholesterolaemic patients. Curr Med Res Opin 2003; 19: 22-7.

29. Senzaki H, Paolocci N, Gluzband YA, et al. Beta-blockade prevents sustained metalloproteinase activation and diastolic stiffening induced by angiotensin II combined with evolving cardiac dysfunction. Circulation Res 2000; 86: 807-15. 